\title{
Quantifying Dry Rubber Content in Latex Solution Using an Ultrasonic Pulse
}

\author{
P. Kerdtongmee, C. Pumdaung, S. Danworaphong \\ School of Science, Walailak University, Thasala, Nakhon Si Thammarat, 80161, Thailand \\ pkerdtongmee@gmail.com
}

\begin{abstract}
The quality of latex solution harvested from a para rubber tree is determined by the amount of dry rubber content (DRC). In this work, we propose the use of an ultrasonic pulse for quantifying the DRC in latex solution. Fresh latex solutions are acquired locally from different regions in the south of Thailand. The DRC of the solutions is evaluated for calibration purposes by the standard technique as recommended in ISO126:2005. Along with the calibration experiment, the ultrasonic pulse experiment is performed on the same set of the solutions in cylindrical tubes of different lengths. The ultrasonic pulse transverse longitudinally through the tubes which are fully contained with the latex solutions. Ultrasonic speeds and spatial attenuations for different dry rubber contents can then be obtained. Our results reveal that the ultrasonic speed and spatial attenuation are linearly proportional to the amount of dry rubber content in latex solution. Using the empirical relationship between the spatial attenuation and the DRC, we can predict the DRC with the accuracy comparable to that of the microwave-drying technique. Given the size of the tube, our setup is relatively small and can be portable.
\end{abstract}

Keywords: Dry rubber content, ultrasonic attenuation coefficient, latex solution.

\section{INTRODUCTION}

$\mathrm{N}$ ATURAL RUBBER becomes a major agriculture product and plays a vital role in the production of tires, medical gloves, and other rubber-based products. The rubber is suspended in an aqueous serum which is called "latex" [1]. The latex is the raw product of Hevea brasiliensis found mainly in the rain forest in the tropical region. The components of freshly collected latex are rubber particles (cis-1, poly isoprene), proteins, water, resins, sugars, and inorganic particles, forming a colloidal system as shown in Fig.1.(a). In general, latex contains about 30$40 \%$ of rubber particles and $55-65 \%$ of water. However, fresh latex shows $15-45 \%$ of rubber hydrocarbon and about $2-4 \%$ of non-rubber ingredients [2]. Latex is usually sold either in the form of dry rubber sheet or concentrated rubber solution. The price of the field latex depends solely on dry rubber content (DRC). Therefore, it is essential to know the amount of DRC in the latex before trading. The dry rubber content is defined as the mass in grams of rubber present in $100 \mathrm{~g}$ of latex.

After microwave drying, the dry rubber content is seen in Fig.1.(b). The chemical structure of the rubber is present in Fig.1.(c). Finally, the scanning electron micrograph of dry rubber is displayed in Fig.1.(d).

There are various methods that have been employed for determining the DRC. It is possible to categorize them into two types: those using mechanical responses such as viscosity and titration analysis, and those relying on electromagnetic wave responses such as low resolution NMR pulsing and microwave dielectric measurement [2, 4]. These techniques require complex tools and specific expertise for measurements and are rather cumbersome for field works. For practical uses, especially in a small trading market, there are three ways of measuring DRC, standard drying ISO126:2005 [5], specific gravity measurement (Metrolac device) [6] and microwave-assisted drying [7].
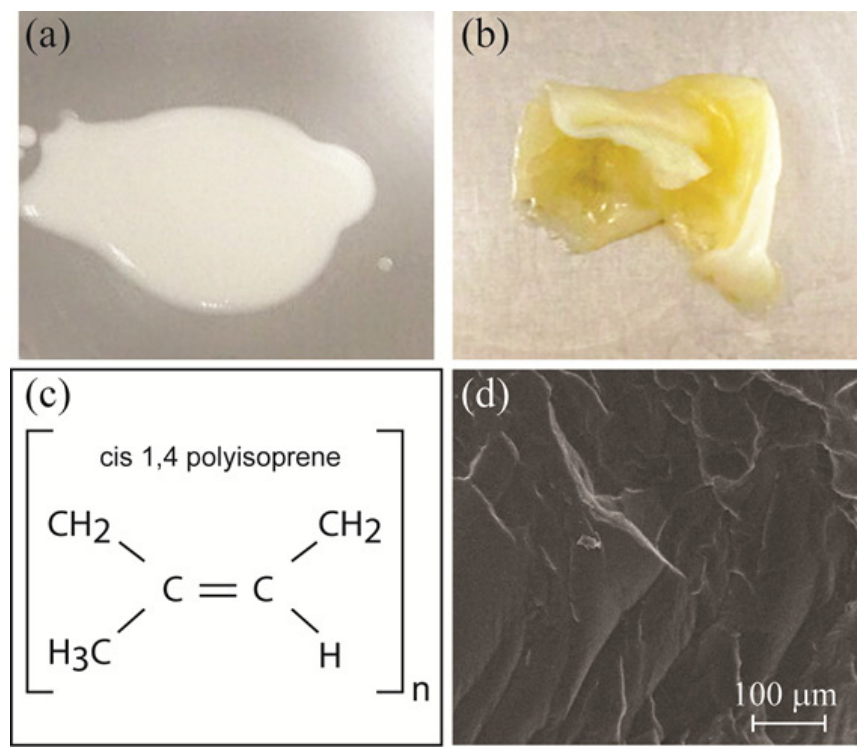

Fig.1. (a) Typical latex solution,

(b) microwave-dried rubber (c) chemical structure of natural rubber [1], and (d) Scanning electron micrograph of dry latex.

The most acceptable method is the procedure illustrated in the ISO126:2005; however, it requires a prolonged period of time, approximately over 12 hours.

Ultrasonic waves have widely been used in the field of non-destructive measurements. The underlying principle for this technique is the variation of ultrasonic speed and attenuation with respect to physical properties of the materials. In liquids and colloids, the wave speed and attenuation characteristics depend on various parameters such as density, viscosity, temperature, and particle size. Therefore, it is possible to derive physical information from changes affecting the speed and attenuation, e.g., monitoring defects in materials [8-13] and determining the 
concentration and particle size distribution of colloidal solutions [14-16]. In this work, a technique based on ultrasonic attenuation is employed to measure the DRC in latex solution. Additionally, we also explore the relationship between ultrasonic speed and the DRC.

Our setup is built upon generic components that can be found in local hardware stores at relatively low cost. Given its size and simplicity, the system could practically be used as a portable DRC meter when the electrical unit, i.e. ultrasonic driver and receiver, as well as the processing unit, are replaced by a microcontroller system. Besides, our results indicate that the system can be used for measuring the DRC with competitive accuracy as compared to those determined by microwave-drying and Metrolac. Also, our system is superior to others in terms of time consumption for each measurement.

\section{SUBJECT AND METHODS}

The ultrasonic attenuation is the decrease of wave amplitude in terms of traveling distance. Referred to [17], if only one ultrasonic pulse is allowed to travel in an ultrasonic cell, the expression for the attenuated pulse, neglecting echoes, can be readily written as

$$
A_{x}=A_{0} e^{-\alpha x}
$$

where $A_{x}$ is the wave amplitude after it has traveled the distance $x, A_{0}$ is the initial amplitude, $\alpha$ is the total attenuation coefficient which may possibly comprise viscous, thermal, scattering and relaxation losses, excluding the classical absorption due to low frequency limit [18]. The scattering effects can be negligible, if the wavelength of the ultrasonic wave is much greater than molecular sizes, [1920]. In this paper, the latex solution is assumed to be homogeneous, given that the wavelength of the ultrasonic waves is about $4 \mathrm{~cm}$, so that the speed is constant along the ultrasonic path. The ultrasonic wave is assumed to be plane; therefore the dispersive effects are negligible [17, 19]. All measurements are carried out at $25^{\circ} \mathrm{C}$.

An ultrasonic cell is constructed to be a cylinder with adjustable length; sometimes it is called "variable path length geometry" [15-16]. The cell is made of acrylic that is designed to work horizontally as shown in Fig.2.(a). Two identical ultrasonic transducers, used for car parking sensors operating at $40 \mathrm{kHz}$, are used as transmitter and receiver. They are carefully aligned so that both ends of the ultrasonic tube share a central line. The transducers are directly in contact with the latex solution. The space between the transducers and the tube wall was sealed by ultrasonic absorber made from silicone. Two holes of 3-mm diameter are drilled on the tube, located $1.00 \mathrm{~cm}$ from the terminal plates, for the introduction of latex solution into the cell. An inner radius of the cell is fixed at $1.90 \mathrm{~cm}$. Four tubes, as shown in Fig.2.(b), are used for length extension for the original cell. With the addition of path-length adjustment tubes, Fig.2.(c), the ultrasonic cell has five working distances, $7.50,10.20,12.90,15.80$ and $17.10 \mathrm{~cm}$. The experimental setup is displayed in Fig.3.(a).

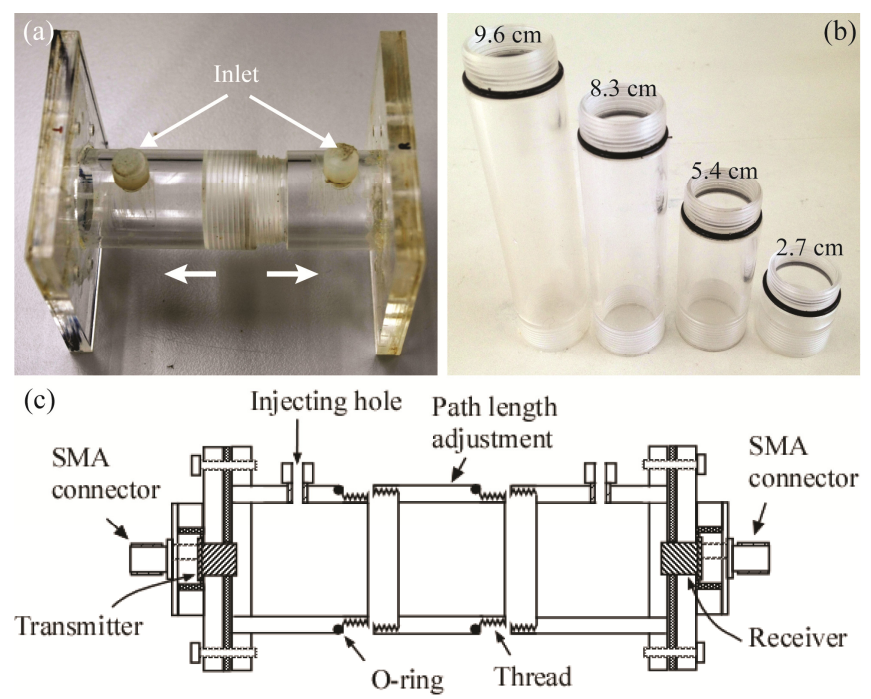

Fig.2. (a) Ultrasonic cell, (b) Tubes with threaded ends for path length adjustment and (c) the detailed drawing of the ultrasonic cell.

(a)

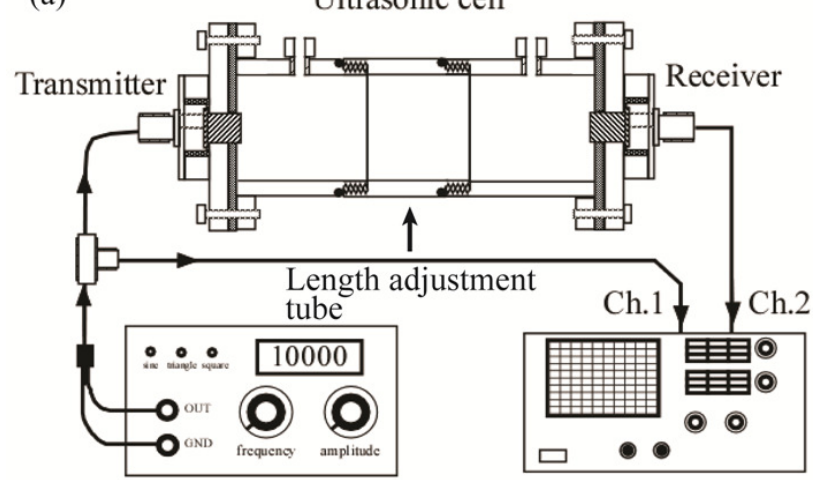

Function generator

Oscilloscope

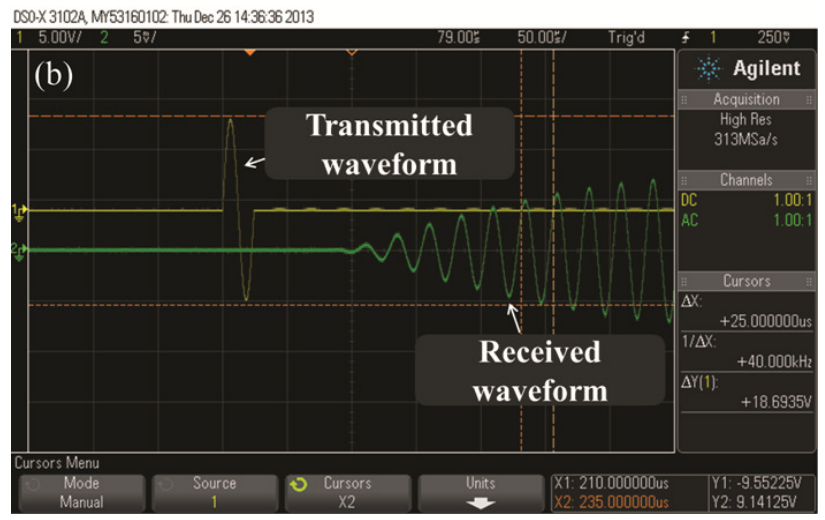

Fig.3. (a) Experimental setup diagram and (b) oscilloscope screen displaying transmitted and received signals.

The length adjustment allows the proper determination of ultrasonic speed and spatial attenuation. A single $40 \mathrm{kHz}$ sine wave is generated from a function generator (HP33120A) as shown in Fig.3.(b). The pulse width is $25 \mu \mathrm{s}$ with the repetition rate of $2 \mathrm{~Hz}$. Such pulsing wave is applied to the transmitter and is designated as the initial ultrasonic pulse on an oscilloscope (DSO-X-3102A). The 
received signal from the other transducer is recorded as the attenuated ultrasonic signal.

For the ultrasonic speed measurement, the latex samples of various DRCs are introduced to the ultrasonic cell of different lengths. Using a simple formula, $x=v t$, where $x$ is the length, $v$ is the ultrasonic speed, and $t$ is the time of flight of the ultrasonic wave, it is therefore possible to evaluate the ultrasonic speed for different DRCs by linear regression analysis. Similarly, all tubes, varying $x$, are employed in order to determine the spatial attenuation, $\alpha$, for each latex sample by using linear regression analysis based on (1).

\section{DETERMINATION OF DRY RUBBER CONTENT}
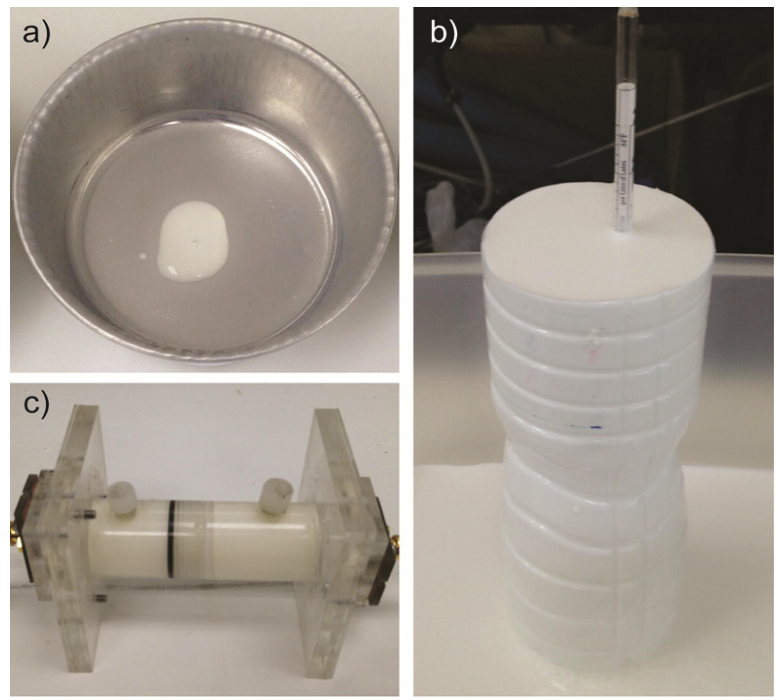

Fig.4. Latex solution for (a) microwave-drying, (b) Metrolac, and (c) ultrasonic techniques.

Latex solutions are obtained directly from rubber farmers. The solutions are then diluted with water to give five unknown concentrations. Each concentration, designated as $\mathrm{S} 1$ to $\mathrm{S} 5$, is divided into four groups. One of them immediately undergoes the procedure as suggested by ISO126:2005 instruction for the determination of DRCs. The process suggests that we have the DRC of $30 \%, 27 \%$, $25 \%, 23 \%$ and $22 \%$. The other two groups simultaneously go through two different tools, microwave drying and Metrolac, as shown in Fig.4.(a) and (b), respectively, for evaluating the DRC. As a result, the DRC values are confirmed, as shown in Fig.5. The DRC values, deduced from the procedure in ISO126-2005, are then used for calibrating the ultrasonic attenuation coefficients. The proposed ultrasonic technique is applied to the last set of latex solutions, as can be seen on the example in Fig.4.(c).

\section{RESULTS AND DISCUSSION}

The average DRCs measured by the Metrolac device and microwave-assisted drying method, as shown in Fig.5., are essentially in agreement with the DRC values obtained from the standard method for all of sample sets. However, slight deviations can still be observed in the measurements, indicated by the error bars. For the Metrolac device, the maximum deviation is about $4.2 \%$ while that of microwaveassisted drying is about $2.8 \%$. The deviation of the Metrolac device is on the average more than that obtained by the microwave-assisted drying because of the motion of reading scale. The deviation from the microwave-assisted drying is probably due to the residual humidity inside the dried rubber and the mass loss on the sample holder.

For the ultrasonic measurement, the latex solution in the last group is introduced to the ultrasonic cell. The pulsing experiment is then performed for all available DRCs, designated as $\mathrm{S} 1$ to $\mathrm{S} 5$, identical to those used in the previous measurements. The time of flight is recorded for different cell lengths allowing the evaluation of ultrasonic wave speed for different DRCs. The speed for each DRC is shown in Fig.6.

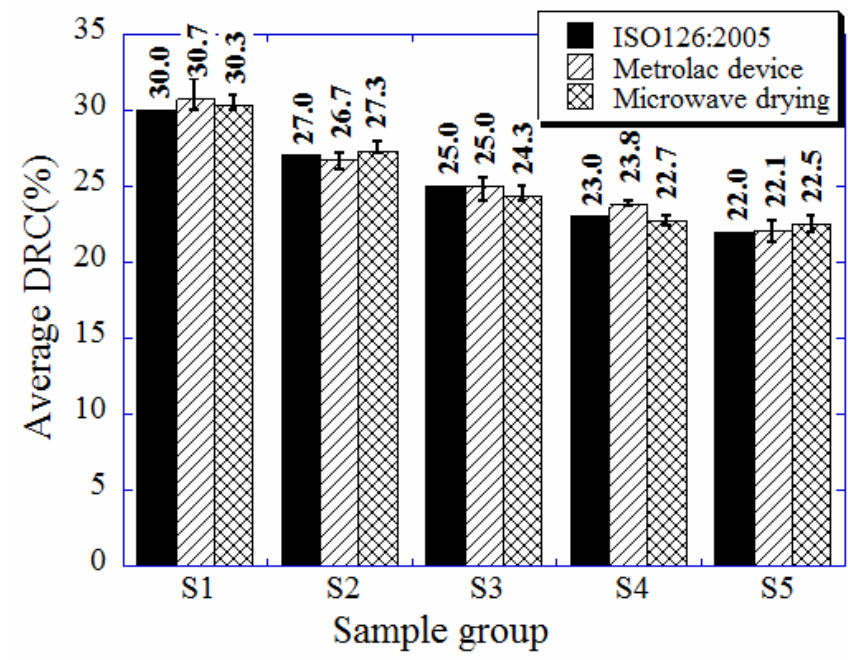

Fig.5. Deviations of DRC from Metrolac device and microwaveassisted drying.

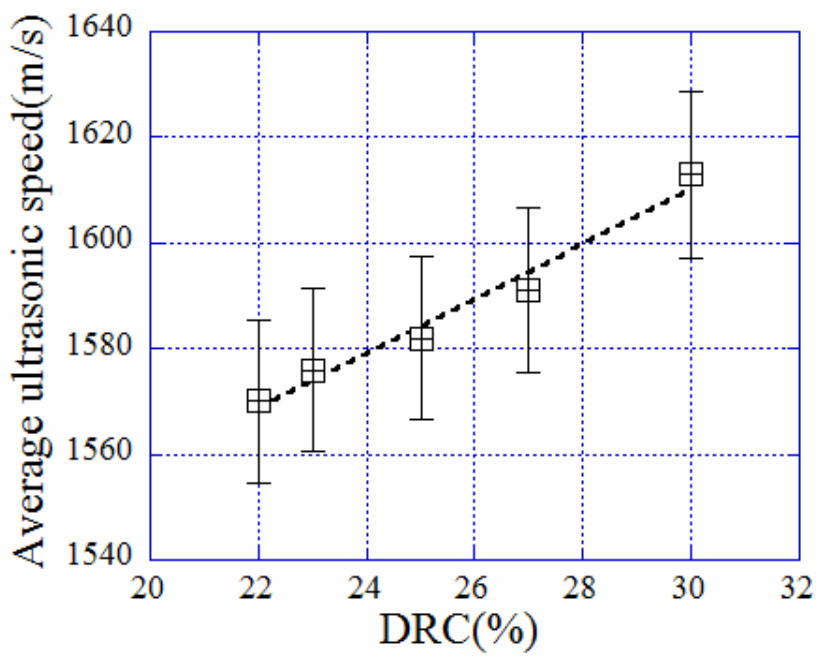

Fig.6. Relationship between the ultrasonic speed and the dry rubber content.

It is apparent that the ultrasonic celerity is linearly proportional to the DRC value. The ultrasonic wave speed varies between $1570-1610 \mathrm{~m} / \mathrm{s}$, corresponding to the average ultrasonic wavelength of $\sim 4 \mathrm{~cm}$, for the DRC of $22-30 \%$. 
The deviation of the length measurement for the tubes by a tape measure yields $1 \mathrm{~mm}$ of deviation which accounts for the variation in the ultrasonic speed of $\sim 15.4 \mathrm{~m} / \mathrm{s}$.

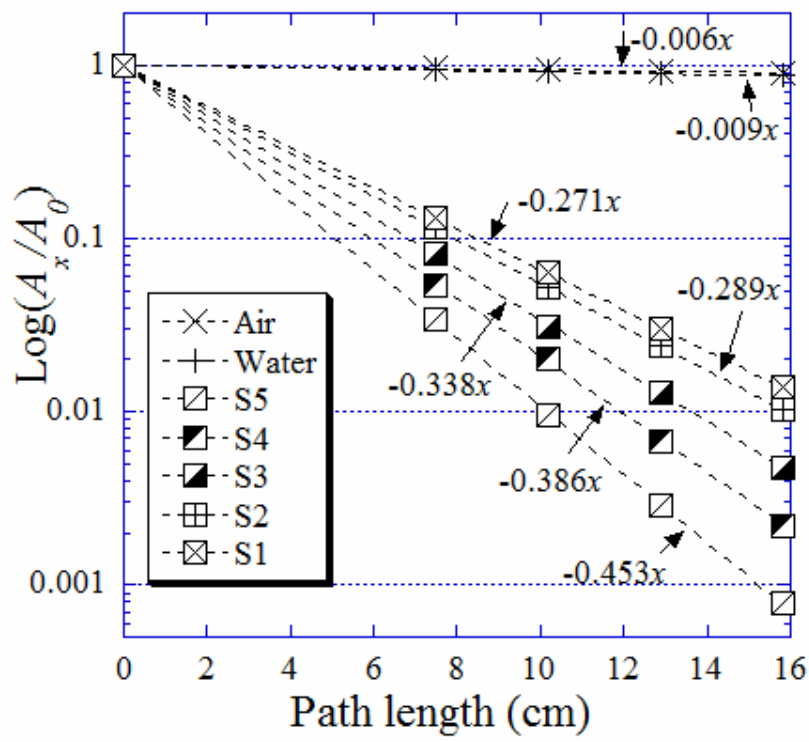

Fig.7. $\log \left(A_{x} / A_{0}\right)$ in terms of path lengths for air $(\times)$ and water $(+)$ and for S5-S1 $(\boldsymbol{\nabla}, \boldsymbol{\Delta}, \boldsymbol{\nabla}, \mathbf{⿴}$ and, $\boldsymbol{\nabla}$, respectively). The dashed lines are the fitting line using (2) as a model. The slopes yield the spatial attenuation coefficients of the dedicated samples.

The spatial attenuations of the ultrasonic wave for S1 to S5 as well as for air are investigated by measuring the decrease of wave amplitude with respect to the distance $x$. The decline of the logarithm of wave amplitude ratio, $A_{x} / A_{0}$, depends on the rubber concentration or DRC as shown in Fig.7. The attenuation can be determined by linearly fitting the logarithm of the ratios in terms of the tube lengths to (1), where the decay factor, $\alpha$, represents the attenuation coefficient. The fitting results provide that $\alpha$ 's are 0.453 , $0.386,0.338,0.289$, and $0.271 \mathrm{~cm}^{-1}$ for $\mathrm{S} 1$ to S5 and 0.009 and $0.006 \mathrm{~cm}^{-1}$ for water and air, respectively. The values of $\alpha$ for air and water are comparable to those seen in [17]. From Fig.7., we find that the ultrasonic method results in small deviation in the logarithm of the amplitude ratio from the fitting result depending on ultrasonic path length $(x)$. Such deviation can be respectively presented for each path length to be $2.7 \%, 3.4 \%, 2.5 \%, 2.3 \%$ and $1.6 \%$ for $\mathrm{x}=$ $7.50,10.20,12.90,15.80$ and $17.10 \mathrm{~cm}$. In terms of sample concentrations, the deviation for $\mathrm{S} 1-\mathrm{S} 5$ is $1.5 \%, 2.3 \%$, $2.0 \%, 0.7 \%$ and $0.85 \%$, respectively.

The attenuation with respect to the standard DRC is displayed in Fig.8. The relation is linearly dependent and can be mathematically expressed as

$$
\% \mathrm{DRC}=43.39 \alpha+10.31
$$

where $\alpha$ has the dimension of $\mathrm{cm}^{-1}$ and its deviation is about $2.3 \%$ yielding the same variance for the values of DRC.

Equation (2) is then used to evaluate the DRC of latex solution from unknown regions. The measurement is performed using the ultrasonic cell with $12.9 \mathrm{~cm}$ path length. The results are displayed in Table 1., where two other techniques are also used to provide the DRCs for comparison purposes. It is clear that our technique shows comparable results to those of the other two methods. The proposed technique can therefore be interchangeably employed for the DRC measurement for practical fieldwork.

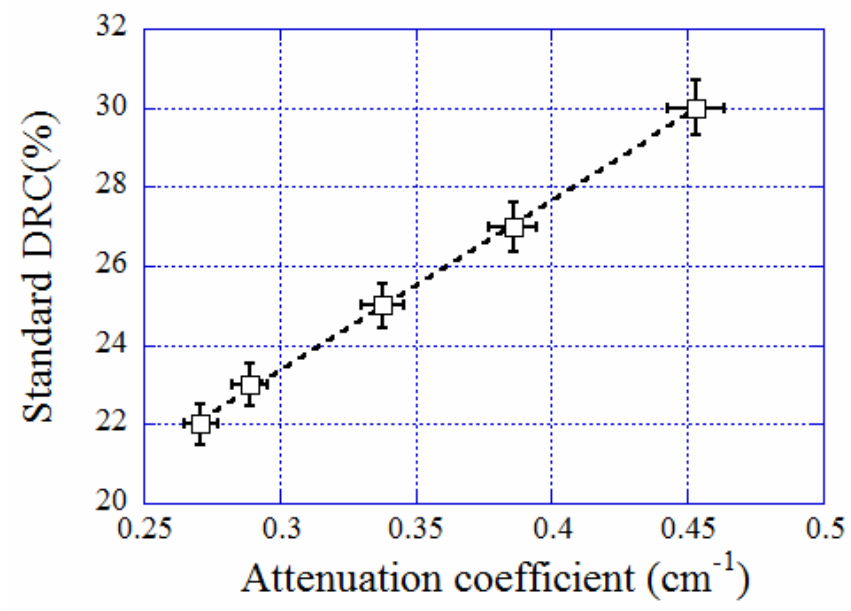

Fig.8. Linear relationship between the DRC's and the attenuation coefficients.

Table 1. Attenuation coefficients and the DRC of unknown latex solutions, resulting from (2), in comparison with those of microwave drying and Metrolac techniques.

\begin{tabular}{|c|c|c|c|c|}
\hline \multirow{2}{*}{$\begin{array}{c}\text { Test } \\
\text { No. }\end{array}$} & $\begin{array}{c}\text { Attenuation } \\
\text { Coefficient }(\alpha), \\
\left(\mathrm{cm}^{-1}\right)\end{array}$ & \multicolumn{3}{|c|}{\begin{tabular}{c} 
Average DRC Percentage \\
\cline { 3 - 5 }
\end{tabular}} \\
\cline { 3 - 5 } & 0.35 & 25.67 & 25.00 & 25.46 \\
\hline 2 & 0.15 & 16.67 & - & 16.70 \\
\hline 3 & 0.37 & 26.33 & 25.57 & 25.00 \\
\hline 4 & 0.48 & 31.00 & 31.06 & 30.00 \\
\hline 5 & 0.32 & 24.67 & 24.25 & 24.00 \\
\hline 6 & 0.25 & 20.00 & 20.00 & 20.95 \\
\hline
\end{tabular}

\section{CONCLUSION}

Correlation techniques such as a Metrolac device and microwave-assisted drying are useful for DRC measurement, however, they show fluctuations in the measurement, within $4.2 \%$ for Metrolac and 2.8\% for microwave-assisted drying. Using the proposed ultrasonic wave, we show that both ultrasonic speed and attenuation are linearly proportional to DRC in the latex solution, providing two alternative ways that can be acquired simultaneously in one measurement. They can be used to confirm one another. The deviation for the attenuation measurement yields the value of $2.3 \%$. The ultrasonic technique is also superior to the other two techniques in certain aspects, i.e., the amount of the latex solution used as compared to that of Metrolac and the time consumption as compared to microwave drying. In addition, the proposed technique can potentially be made portable if its electrical components are embedded on an integrated circuit board.

\section{ACKNOWLEDGEMENT}

The authors gratefully acknowledge the Institute of Research and Development (IRD), Walailak University, Thailand for WU56702 research fund. 


\section{REFERENCES}

[1] Matthan, R.K. (1998). Rubber Engineering. New Delhi, India: Tata McGraw-Hill.

[2] Jayanthy, T., Sankaranarayanan, P.E. (2005). Measurement of dry rubber content in latex using microwave technique. Measurement Science Review, 5 (3), 50-54.

[3] Kumar, R.R. (2009). Design and development of instrumentation systems to determine the dry rubber content in natural rubber latex. Doctoral dissertation. Cochin University of Science and Technology, Kerala, India.

[4] Ansarudin, F., Abbas, Z., Hassan, J., Yahaya, N.Z., Ismail, M.A. (2012). A simple insulated monopole sensor technique for determination of moisture content in hevea rubber latex. Measurement Science Review, 12 (6), 249-254.

[5] International Organization for Standardization. (2005). Natural rubber latex concentrate - Determination of dry rubber content. ISO126:2005.

[6] Gils, G.E.V. (1940). The specific gravity of rubber and of serum in Hevea latex. Rubber Chemistry and Technology, 13 (2), 422-429.

[7] Khalid, K.B., Wahab, Z.B.A., Kasmani, A.R. (1988). Microwave drying of Hevea rubber latex and total solid content determination. Pertanika, 11 (2), 289297.

[8] Murai, Y., Ohta, S., Shigetomi, A., Tasaka, Y., Takeda, Y. (2009). Development of an ultrasonic void fraction profiler. Measurement Science and Technology, 20 (11), 1-13.

[9] Motozawa, M., Iizuka, Y., Sawada, T. (2008). Experimental measurements of ultrasonic propagation velocity and attenuation in a magnetic fluid. Journal of Physics: Condensed Matter, 20 (20), 1-5.

[10] Mather, M.L., Charles, P.H., Baldock, C. (2003). Measurement of ultrasonic attenuation coefficient in polymer gel dosimeters. Physics in Medicine and Biology, 48 (20), N269-N275.
[11] Carson, G., Mulholland, A.J., Nordon, A., Tramontana, M., Gachagan, A., Hayward, G. (2008). Particle sizing using passive ultrasonic measurement of particle-wall impact vibrations. Journal of Sound and Vibration, 317 (1-2), 142-157.

[12] Stor-Pellinen, J., Hæggstrom, E., Karppinen, T., Luukkala, M. (2002). Air-coupled ultrasonic transmission measurement through paper during wetting. Measurement Science and Technology, 13 (5), 770-774.

[13] Seco, F., Jimenez, A.R., Castillo, M.D. (2006). Air coupled ultrasonic detection of surface defects in food cans. Measurement Science and Technology, 17 (6), 1409-1416.

[14] Challis, R.E., Povey, M.J.W., Mather, M.L., Holmes, A.K. (2005). Ultrasound techniques for characterizing colloidal dispersions. Reports on Progress in Physics, 68 (7), 1541-1637.

[15] McClements, D.J. (2006). Ultrasonic measurements in particle size analysis. In Encyclopedia of Analytical Chemistry. John Wiley \& Sons, 1-8.

[16] Eggers, F., Kaatze, U. (1996). Broad-band ultrasonic measurement techniques for liquids. Measurement Science and Technology, 7 (1), 1-19.

[17] Shields, F.D., Bass, H.E., Bolen L.N. (1977). Tube method of sound-absorption measurement extended to frequency far above cutoff. The Journal of the Acoustical Society of America, 62 (2), 346-353.

[18] Hauptmann, P., Hoppe, N., Puttmer, A. (2002). Application of ultrasonic sensors in the process industry. Measurement Science and Technology, 13 (8), R73-R83.

[19] Leydier, A., Mathieu, J., Despaux, G. (2009). The two coupling fluids method for ultrasonic velocity measurement. Application to biological tissues. Measurement Science and Technology, 20 (9), 1-7.

[20] Hendee, W.R., Ritenour, E.R. (2002). Medical Imaging Physics, $5^{\text {th }}$ ed. Wiley.

Received January 03, 2014. Accepted September 30, 2014. 